\title{
Design and Fabrication of 2-T Rotating Halbach Magnet for Magnetic Refrigerator
}

\author{
Kwon Sang Ryu ${ }^{1,2 *}$, Seung Hoon Nahm ${ }^{1,2}$, Jae Kap Jung ${ }^{1,2}$, and Seung-Wook Baek ${ }^{1}$ \\ ${ }^{1}$ Korea Research Institute of Standards and Science (KRISS), Daejeon 34113, Korea \\ ${ }^{2}$ Science of Measurement, University of Science \& Technology (UST), Daejeon 34113, Korea
}

(Received 3 January 2017, Received in final form 16 February 2017, Accepted 20 February 2017)

\begin{abstract}
This paper reports simulation and evaluation of the magnetic flux density (MFD) of a rotating Halbach magnet (RHM) composed of a rotating inner Halbach cylinder (HC) and a fixed outer HC. We first chose the dimension of a single $\mathrm{HC}$, then determined the dimensions of an inner and outer $\mathrm{HC}$ to get $\mathrm{MFD}=2 \mathrm{~T}$. Simulation results were used to guide fabrication of an RHM; its magnetic field in the central axis direction agreed well with simulated values.
\end{abstract}

Keywords : Rare-earth magnet, Halbach cylinder (HC), Rotating Halbach magnet (RHM), Finite element method (FEM), Magnetic flux density (MFD)

\section{Introduction}

The magnetocaloric effect determines the efficiency of a magnetic refrigerator. When the refrigerant magnetizes or demagnetizes, the magnetocaloric effect raises or lowers its adiabatic temperature [1]. These refrigeration processes are very similar those that occur in a conventional refrigeration by cyclic compression and expansion of a gas [2]. The entropy change of numerous magnetocaloric materials scales as a power law with an exponent that generally $\sim 2 / 3$ [3-5]. As a source of the magnetic field for the magnetic refrigerator, permanent magnets are preferred because they do not require power to generate a magnetic field, so and only mechanical energy is required to move the magnet or the active material $[5,6]$. A source with magnetic flux density (MFD) of $1 \mathrm{~T}$ or $2 \mathrm{~T}$ in an acceptable air-gap volume is suitable for use in compact systems that require a few kilowatts of power [6,7]. To obtain this MFD, structure configuration must be optimized.

The use of high-energy rare-earth magnets in the conventional technology can improve greatly the efficiency of the devices, and are of central importance for magnetic refrigeration [8]. Rare earths are much more expensive than iron, cobalt and nickel, so a rare-earth magnet array

(C)The Korean Magnetics Society. All rights reserved.

*Corresponding author: Tel: +82-42-868-5164

Fax:+82-42-868-5635, e-mail: ryuks@kriss.re.kr should have the highest magnetic field for a given size and weight of permanent magnet material [9]. The magnetic field strength can be increased by using a Halbach cylinder (HC) made of a rare earth magnet and shielded by soft magnetic materials $[10,11]$.

In this work, we report the design and fabrication of a rotating Halbach magnet (RHM) that produces MFD $=2$ $\mathrm{T}$. We first chose the dimension of a single $\mathrm{HC}$, then determined the dimensions of the inner and outer HCs that yield MFD $=2 \mathrm{~T}$. We used simulation to guide fabrication of an RHM; its measured fields agreed well with simulated values.

\section{Design and Fabrication of RHM}

The RHM considered (Fig. 1(a), (b)) consists of twelve permanent magnet segments that enclose an air gap (Fig. 1(b)). Access to the magnetic field in the air gap is possible only from the right side of the RHM. The magnetic field in the air gap is greatest when the magnetizations of two HCs are in same direction, but smallest when these directions are opposite.

The electromagnetic field simulation used the finite element method (FEM) on ANSYS Maxwell v15 3D software [12], with Magnetostatic as the solver. The governing equations for these processes are:

$$
\nabla \times \frac{1}{\sigma} \nabla \times \vec{H}+\frac{\partial \vec{B}}{\partial t}=0
$$




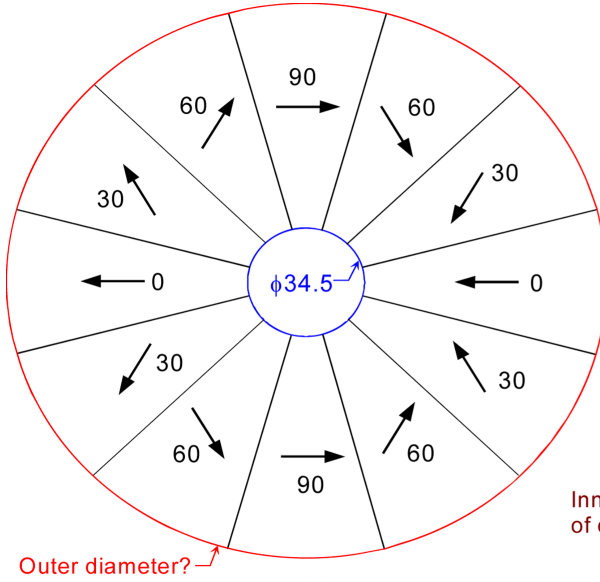

(a)

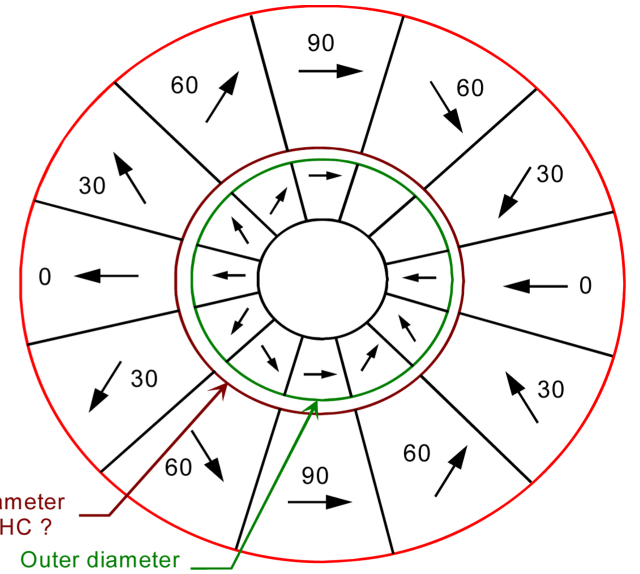

(b)

Fig. 1. (Color online) Model for simulating the MFD of the RHM. (a) Model for calculating the outer diameter of the single HC. (b) Model for calculating the inner diameter of the outer HC and the outer diameter of the inner HC. Arrows show magnetization.

$$
\begin{aligned}
& \nabla \cdot \vec{B}=0 \\
& -\nabla \cdot\left(\varepsilon \nabla \frac{\partial \Phi}{\partial t}\right)-\nabla \cdot(\sigma \nabla \Phi)=0
\end{aligned}
$$

where $\sigma[\mathrm{S} / \mathrm{m}]$ is the conductivity, $\vec{H}[\mathrm{~A} / \mathrm{m}]$ is the magnetic field intensity, $\vec{B}[\mathrm{~T}]$ is the MFD, $\varepsilon[\mathrm{F} / \mathrm{m}]$ is permittivity, and $\Phi[\mathrm{Wb}]$ is the magnetic flux.

The 2-T RHM (Fig. 1(b)) is composed an inner HC rotor and an outer $\mathrm{HC}$ stator. The permanent magnet was a neodymium magnet $\mathrm{N} 50 \mathrm{M}$ with coercivity $=1,042 \mathrm{~A} /$ $\mathrm{m}$, and remanence $=1.4 \mathrm{~T}$. To produce $\mathrm{MFD}=2 \mathrm{~T}$, the outer diameter of the single $\mathrm{HC}$ must be determined first (Fig. 1(a)). The single HC was composed of 12 pieces, each magnetized to a different direction (Fig. 1(a), arrows). Each HC was $200 \mathrm{~mm}$ long; the inner diameter in which the refrigerant material is established was chosen to be $34.5 \mathrm{~mm}$. Along the axial direction FEM simulation (Fig. 2(a)) suggest that MFD $>2 \mathrm{~T}$ when the outer radius is $>90 \mathrm{~mm}$. The MFD was proportioned to the outer radius $r_{o}[\mathrm{~mm}]$ of the single $\mathrm{HC}$ (Fig. 2(b)), as

$$
B=1.01+0.012 \times r_{o}
$$

We set $r_{o}=97 \mathrm{~mm}$ because the MFD may leak through at air gap between the inner and outer HC. At this time, MFD was $\sim 2.15 \mathrm{~T}$.

When determining the outer diameter of the inner $\mathrm{HC}$, the RHM must have maximum MFD $>2 \mathrm{~T}$ and minimum $\mathrm{MFD} \approx 0 \mathrm{~T}$ (Fig. 3, 4). The MFD was $>2 \mathrm{~T}$ when the inner $\mathrm{HC}$ had $r_{o}>30.62 \mathrm{~mm}$ (Fig. 3). The profiles of minimum MFD are more complex (Fig. 4(a)) than those of maximum MFD. The peaks of the maximum MFD do not only appear at center, but also at both ends of $\mathrm{HC}$. The MFD at the axial core decreased as $r_{o}$ of the inner $\mathrm{HC}$ increased, until $r_{o}=38.62 \mathrm{~mm}$, then increased as $r_{o}$

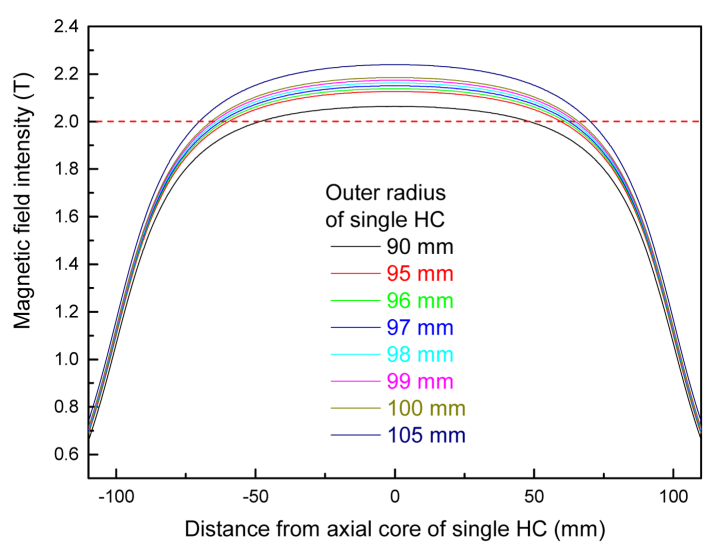

(a)

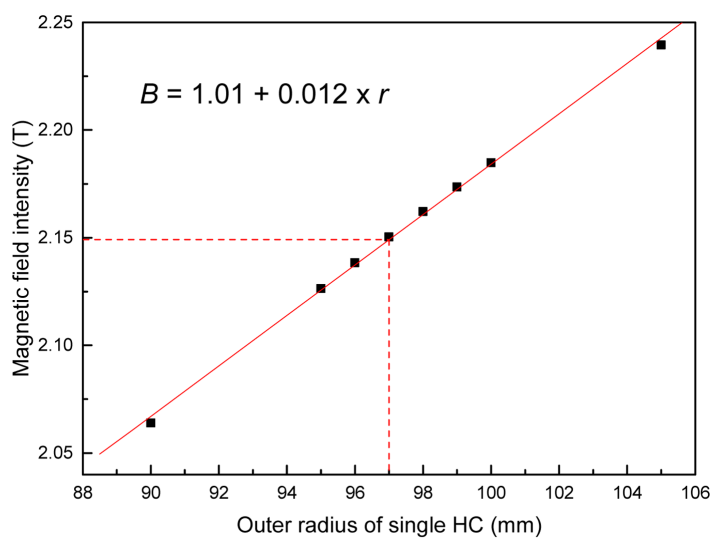

(b)

Fig. 2. (Color online) (a) Profiles for simulating magnetic field of axial direction according to the outer radius of the single HC. (b) Result of the least square fitting at the axial core of single HC. 


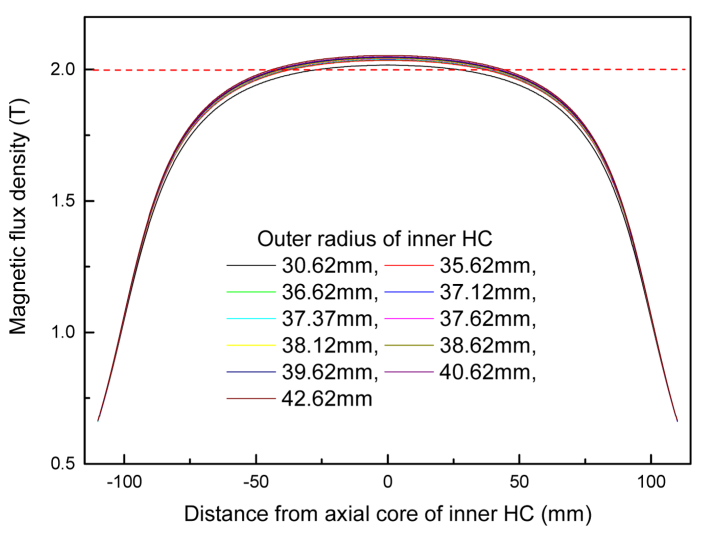

(a)

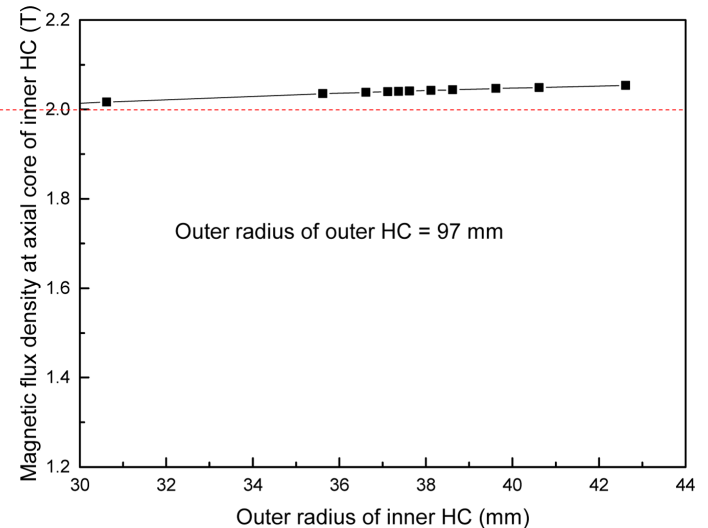

(b)

Fig. 3. (Color online) (a) Profiles for simulating magnetic field of axial direction of HM according to the outer radius of the inner $\mathrm{HC}$ when that the magnetizations of the inner and outer $\mathrm{HC}$ are same direction. (b) Result of the least square fitting at the axial core of the HM.

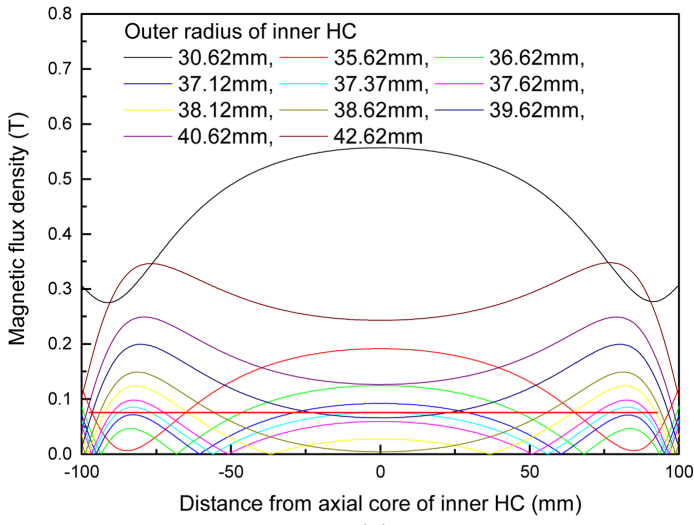

(a)

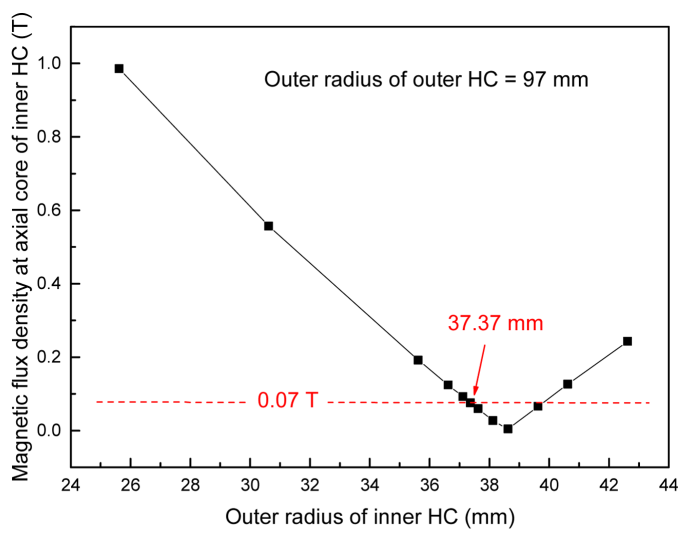

(b)

Fig. 4. (Color online) (a) Profiles for simulating magnetic field of axial direction of HM according to the outer radius of the inner $\mathrm{HC}$ when that the magnetizations of the inner and outer $\mathrm{HC}$ are opposite direction. (b) Result of the least square fitting at the axial core of the HM.

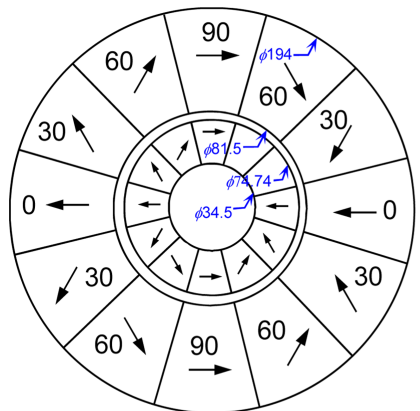

(a)

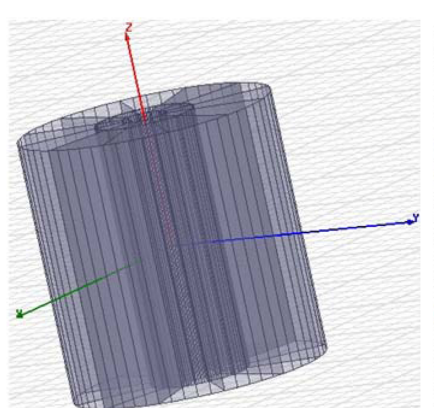

(b)

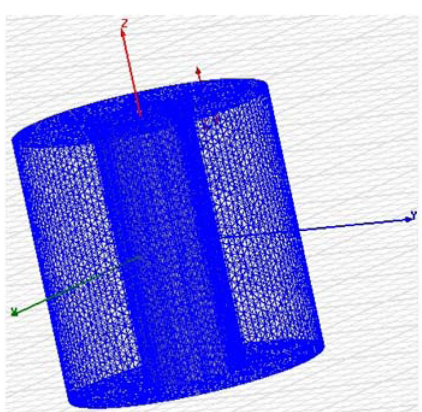

(c)

Fig. 5. (Color online) (a) Determined dimension, (b) the estimated model for simulating the magnetic field, and (c) the mesh configuration of RHM.

increased further. We chose $r_{o}=37.37 \mathrm{~mm}$ (Fig. 5(a)) by considering the three peaks, so RHM had minimum MFD $=0.07 \mathrm{~T}$.

The chosen dimensions of RHM are as follows. The
$\mathrm{HC}$ had outer diameter $=194 \mathrm{~mm}$ and inner diameter $=$ $81.5 \mathrm{~mm}$. The inner $\mathrm{HC}$ had outer diameter $=74.74 \mathrm{~mm}$ and inner diameter $=34.5 \mathrm{~mm}$. The air gap between the inner $\mathrm{HC}$ and outer $\mathrm{HC}$ was $3.38 \mathrm{~mm}$. The RHM was 


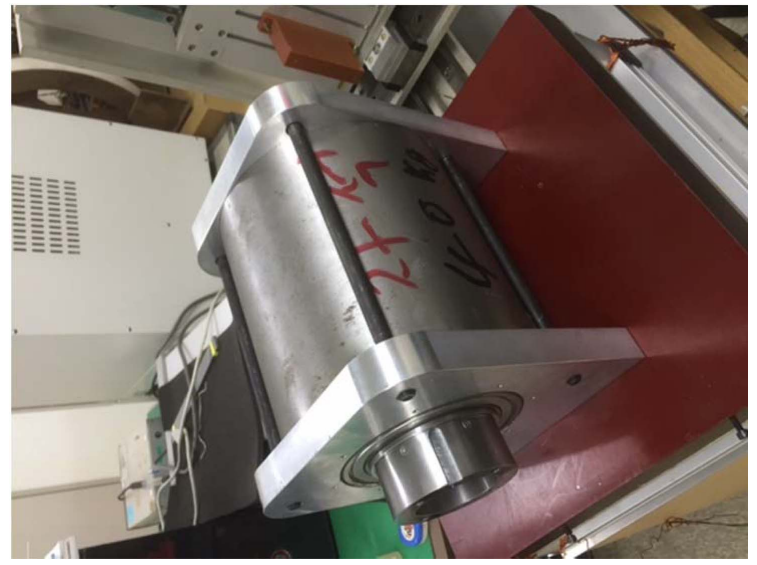

Fig. 6. (Color online) Photograph of the fabricated RHM.

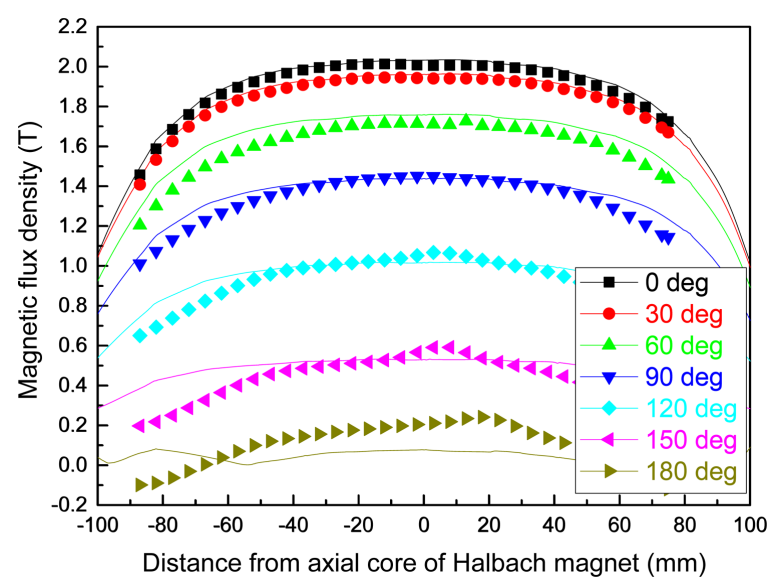

Fig. 7. (Color online) MFD profiles of the RHM. The lines are simulated MFD, and symbols are measured one.

simulated using a mesh composed of $\sim 1,252,800$ tetrahedra (Fig. 5(c)). The RHM was manufactured at the Baotou Research Institute of Rare Earth in China on the basis of the simulation [13].

\section{Results and Discussion}

The RHM (Fig. 6) was fabricated in accordance with simulation results, and the MFD of the central axis was measured using a Gaussmeter (F.W. Bell 8030) with flattype Hall probe (STF81-0404-10) by rotating the inner $\mathrm{HC}$ in increments of $30^{\circ}$. The measured results were compared to the simulated results. When the magnetizations of inner and outer $\mathrm{HC}$ are in the same direction, the MFD should be greatest; the maximum values MFD were of $2.03 \mathrm{~T}$ in the simulation and $2.01 \mathrm{~T}$ in measurement. The simulated and measured MFDs of the RHM agreed well at $0^{\circ}$ and $30^{\circ}$, but less well at $150^{\circ}$ and $180^{\circ}$ (Fig. 7).

\section{Conclusion}

A strong magnetic field is important in a magnetic refrigerator, because the magnetocaloric effect at the Curie temperature increases as $\mathrm{MFD}^{2 / 3}$. A rotating Halbach magnet (RHM) is composed of an inner Halbach cylinder (HC) rotor and an outer HC stator. We first used FEM to simulate the size of a single $\mathrm{HC}$, then determined the dimensions of the inner $\mathrm{HC}$ to produce $\mathrm{MFD}=2 \mathrm{~T}$. We determined the outer diameter of the single HC to be 194 $\mathrm{mm}$. The inner $\mathrm{HC}$ had inner $=34.5 \mathrm{~mm}$ and outer diameter $=74.74 \mathrm{~mm}$. We used simulation results to guide fabrication of the RHM. The measured MFD agreed well with simulated values.

\section{References}

[1] X. N. Xu, D. W. Lu, G. Q. Yuan, Y. S. Han, and X. Jin, J. Appl. Phys. 95, 6302 (2004).

[2] X. Bohigas, E. Molins, A. Roig, J. Tejada, and X. X. Zhang, IEEE Trans. Magn. 36, 538 (2000).

[3] V. Franco, A. Conde, V. K. Pecharsky, and K. A. Gschneidner Jr, Europhysics Lett. 79, 47009 (2007).

[4] Q. Dong, H. Zhang, J. Sun, B. Shen, and V. Franco, J. Appl. Phys. 103, 116101 (2008).

[5] R. Bjfrk, C. R. H. Bahl, A. Smith, and N. Pryds, Int'l. J. Refrigeration 33, 437 (2010).

[6] F. Allab, A. Kedous-Lebouc, J. P. Yonnet, and J. M. Fourier, Int'l. J. Refrigeration 29, 1340 (2006).

[7] A. Tura and A. Rowe, Int'l. J. Refrigeration 34, 628 (2011).

[8] W. Dai, B. G. Shen, D. X. Li, and Z. X. Gao, J. Magn. Magn. Mater. 218, 25 (2000).

[9] S. J. Lee and D. C. Jiles, IEEE Trans. Magn. 38, 2991 (2002).

[10] U. B. Baek, J. S. Lee, S. C. Yu, and K. S. Ryu, J. Magn. 19, 349 (2014)

[11] K. Halbach, Nucl. Instrum. Methods 1691 (1980).

[12] Ansys inc., http://www.ansys.com.

[13] Baotou Research Institute of Rare Earth, http://www.brire.com. 